\title{
Industrial-Like Vehicle Platforms for Postgraduate Laboratory Courses on Robotics
}

\author{
Pedro J. Navarro, Carlos Fernández, and Pedro Sánchez
}

\begin{abstract}
The interdisciplinary nature of robotics allows mobile robots to be used successfully in a broad range of courses at the postgraduate level and in $\mathbf{P h . D .}$. research. Practical industrial-like mobile robotic demonstrations encourage students and increase their motivation by providing them with learning benefits not achieved with traditional educational robotic platforms. This paper presents VEGO, an industrial-like modular vehicle platform for robotic education with an appropriate infrastructure that has been demonstrated to be very useful at the postgraduate level. Besides learning engineering concepts, in performing industrial-like exercises, students develop valuable skills such as teamwork and the capacity to solve problems similar to those they may encounter in a real industrial environment. The developed infrastructure represents a valuable platform for robotic education that can be used in many different disciplines as a way to demonstrate how to cope with the difficulties and challenges related to the development of industrial infrastructure systems. The platform evaluation proved its ability to inculcate the expected engineering skills. A novel approach is adopted through the use of multidisciplinary and close-to-industrial-reality platforms developed under an incremental approach and using an open and customizable structure.
\end{abstract}

Index Terms-Curriculum issues, multidisciplinary laboratory, postgraduate courses, robotics education, robotized vehicle platforms.

\section{INTRODUCTION}

$\mathbf{O}$ VER the last decade, a growing number of projects and initiatives have been dedicated to new educational technologies in robotics education; some representative examples can be found in [1]-[7]. Various educational platforms have been introduced, with a variety of simulators, remote infrastructures, videos, computer applications, and the like. These resources are usually integrated in the curriculum of universities to upgrade the learning outcomes of their students. Many of these universities have developed sophisticated mobile robot platforms with very different levels of autonomy and capacity. Moreover, this has required the integration of the related engineering fields, such as electrical and mechanical engineering and computer science. Although several very good results have been obtained from the design and implementation of these

Manuscript received May 09, 2012; accepted August 07, 2012. This work was supported in part by the Fundación Séneca of the Murcia Region under Grant 15374/PI/10, the CICYT EXPLORE under Grant TIN2009-08572, and the INNPLANTA SiveLab, Ministry of Science and Innovation, Spain, under Grant INP-2011-0022-PCT-430000-ACT9.

The authors are with the División de Sistemas e Ingeniería Electrónica, Universidad Politécnica de Cartagena, Cartagena 30202, Spain (e-mail: pedro.sanchez@upct.es).

Digital Object Identifier 10.1109/TE.2012.2213090 autonomous mobile robots, less work has been published on multidisciplinary industrial-like robots with capabilities for more complex tasks related to localization, navigation control, exploration, obstacle detection, path planning, and path tracking; similarly, little has been published on familiarizing students with the problems arising from the use of industrial components in robotic systems. This is because the cost of obtaining a more powerful robot is a deterrent in comparison to other low-cost solutions. It is worth noting that LabVIEW Robotics as an interesting platform for the development of mobile robots with industrial-like control systems. In this sense, it is becoming common to use the out-the-box DaNI platform [8].

The aim of the work described here was to design and implement a mobile robot platform that would be useful for the integration of many engineering disciplines and would present the same problems, challenges, and risks that students are sure to find in the industrial context. One of the biggest challenges in this work has been the development of industrial-like platforms with incremental complexity, coupled with the design of an appropriate set of scenarios that allows a suitable hands-on approach to project-based learning (PBL) [9]. PBL incorporates a number of fundamental concepts that the students must learn, understand, and apply in order to complete a project.

The remainder of this paper is structured as follows. Section II considers the background of the selected approach. Section III describes the vehicle platforms the authors selected and developed for the courses. Section IV gives a detailed account of the development of the lab modules. Section V discusses the results obtained and, finally, Section VI draws conclusions.

\section{BACKGROUND}

The "Information and Communications Technologies" Master's of Science (M.Sc.-ICT) program has been offered at the Universidad Politécnica de Cartagena, Cartagena, Spain for the last decade. Full-time students take a whole academic year to complete the program, and part-time students usually complete it in two years. There are two mandatory robotics-related modules available for M.Sc. students: "Robotics and Computer Vision" and "Software Development for Real-Time Systems Design." There are a total of 10 one-semester modules, among them "Advanced Learning on Signal Processing," "Electronic Systems," "Communications Networks," and "Radiocommunication Systems." The students in the two mandatory modules are assessed without exams, based on their daily work during the course and on their final project. The authors are convinced that this type of evaluation motivates students to pursue research on robotics-related areas. The M.Sc.-ICT is oriented to research and is a precursor to Ph.D.-level work; 


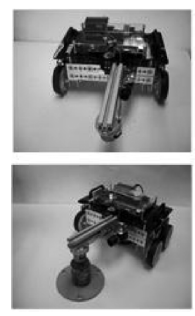

NI Robot (a)

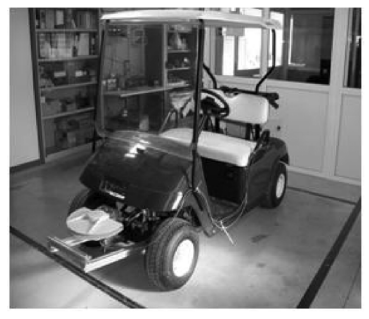

Front view

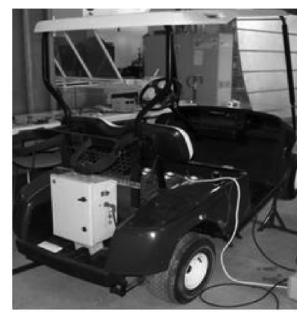

Rear view (b)

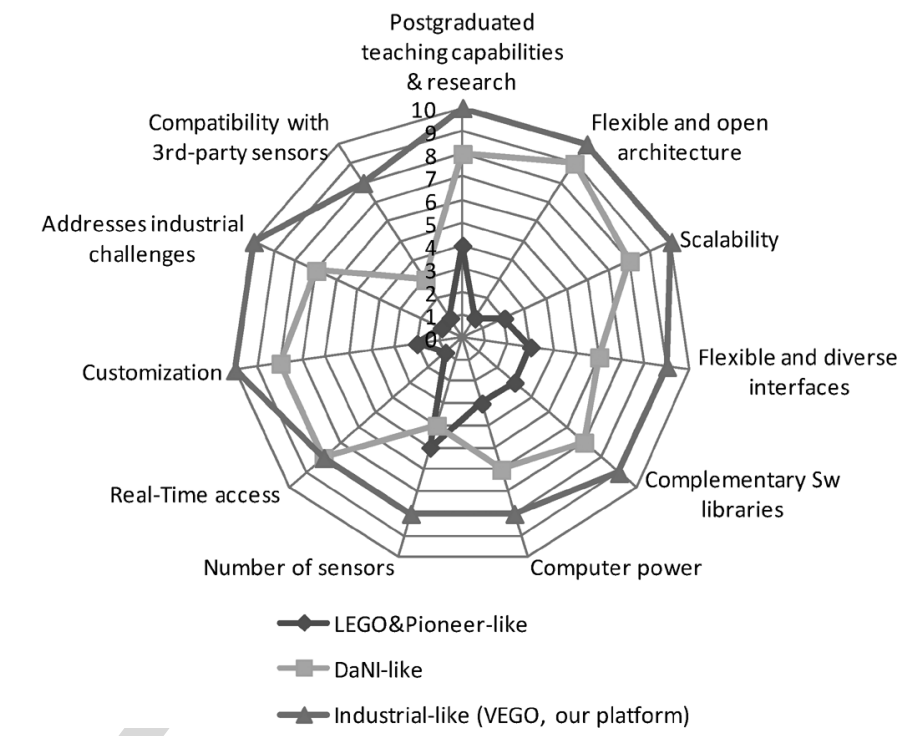

since it was inaugurated, a total of $17 \mathrm{Ph} . \mathrm{D}$. theses have been completed. A total of 145 students finished the M.Sc.-ICT, of whom 35 defended M.Sc. theses related to robotics between the academic years 2006-2007 and 2010-2011. Of these students $53 \%$ had a degree in Telecommunication Engineering, 36\% in Industrial Engineering, and 11\% in different areas like Physics, Mathematics, or Computing Science.

The lectures are conducted mainly by the members of the División de Sistemas e Ingeniería Electrónica (DSIE) research group, who are deeply committed to the industrial applications of robotics. Many benefits follow when a course is delivered by a team with a strong interest in, and research focus on, solving real application problems. The need to use platforms near to field applications in mobile robotics, together with the ability to get mobile robots to react intelligently to their environment, is a highly developed research field that is explored during the course. This research field also provides an ideal application area for applying techniques such as algorithmic problem solving, computer vision, perception, planning systems, fault tolerance, or real-time and embedded systems. Other related work areas include programming languages and computer architectures. In addition to such disciplines within the scope of computer science, robotics is a scientific discipline that also includes the electronics, electrical, and mechanical engineering disciplines.

To cover this wide range of disciplines, the authors' main requirements for teaching and research platforms were: 1) closeness to industrial robotic applications; 2) integration of commercial $\mathrm{Hw} / \mathrm{Sw}$ control modules; 3 ) advanced sensing capabilities; and 4) open and customizable architecture. The following description of the platform shows how those requirements were satisfied.

\section{INDUSTRIAL-Like Vehicle Platforms}

The design of the laboratory was based on mobile robotic platforms characterized by their strong multidisciplinarity, that is, they need contributions from various knowledge areas that offer open architectures and are close to industrial reality. Fig. 1 shows the two platforms used by the students: (a) an out-of-the-box starter kit (the NI LabVIEW Robotics Starter Kit, DaNI), and (b) the robotized golf cart, called VEGO, developed by the authors and described in this paper.

LabVIEW was used as the software design tool since: 1) it provides a comprehensive and scalable platform that spans the design, prototype, and deployment phases, allowing students to focus on robotics without getting distracted by implementation

Fig. 2. Authors' estimation of the possibilities offered by each platform (scale from $0-\min$ to $10-\max$ )

details; and 2) its industrial use is widely extended. LabVIEW Robotics is becoming a powerful robotic development tool because of its ability to integrate with thousands of different devices, proving a uniform programming environment across any $\mathrm{I} / \mathrm{O}$ or sensor type. Students can use the same powerful platform to program controllers ranging from microcontrollers to field-programmable gate arrays (FPGAs); send and receive signals from virtually any sensor and actuator; design and simulate dynamic control systems; and implement an interface to monitor or control the robot remotely. The LabVIEW graphical system design platform encourages accelerated robotic design by providing a uniform platform for all robotic designers [10]; additionally, it makes it easy to program complex robotic applications by providing a high level of abstraction for sensor communication, obstacle avoidance, path planning, kinematics, steering, and more [11].

Both vehicles-DaNI and VEGO - share the same design platform allowing students: 1) to develop control and sensing algorithms independent of the platform; and 2) to validate basic sensing strategies, control, and so on, quickly and without risk, in the DaNI vehicle before immediately implementing them in the VEGO vehicle.

Based on the authors' more than 15 years' experience in teaching robotics, Fig. 2 summarizes the advantages offered by the approach described here in comparison to other commonly found solutions. As observed, LEGO-based and Pioneer-like platforms have some deficiencies that mean that key concepts cannot be treated in depth in advanced robotics courses and distance students from the final industrial reality. DaNI-like platforms are very interesting because they solve most of the problems found in LEGO-based and Pioneer-like platforms, offering a more flexible architecture that allows instructors to assemble systems into a variety of operational units, as well as software libraries of standard modules for advanced tasks (trajectory following, obstacle detection, and so on). However, it is only the industrial-like platform that offers an environment true 
to industrial contexts, with the same challenges and technical difficulties that engineers find in their working environment.

The following provides a detailed description for both platforms, and in particular for VEGO, which was constructed by robotizing a golf cart.

\section{A. NI Single-Board RIO Robot DaNI}

The NI LabVIEW Robotics Starter Kit, shoyn in Fig. 2(a)/Should this be Fig. 1(a) $\overline{\overline{\bar{\nu}}}$ is an out-of-the-box mobile robot platform that features sensors, motors, and NI single-board RIO hardware for embedded control. The controller is a low-cost embedded deployment solution that integrates a real-time processor, reconfigurable FPGA, analog and digital $\mathrm{I} / \mathrm{O}$ on a single board, a module for high-speed control, custom I/O timing, and inline signal processing. The simplicity of this starter kit makes it ideal for teaching robotics and mechatronics concepts, or for developing a robot prototype with LabVIEW Robotics.

Students can easily change the behavior of the robot by developing their own algorithms from the ground up, or by using algorithms built into LabVIEW Robotics software such as path tracking. M.Sc. students use this robotic starter kit equipped with in-kit sensors (ultrasound obstacle detection) to build autonomous robots. The greatest advantage of this platform is its industrial-like open hardware structure and the possibility of teaching the embedded technology with a combination of real-time and on-board FPGA chips to build fully customizable solutions, while students avoid problems associated with mechatronics and control design in the initial learning stages. On the other hand, this platform does not give full freedom to experiment in the areas of integration of third-party sensors (laser range finders, global positioning systems, vision cameras, etc.), control of devices (motors, actuators, etc.), or the integration of industrial buses for communication.

\section{B. Robotized Golf Cart-VEGO}

Once students get used to developing applications for mobile robotics, they start to work with VEGO, a platform close to industrial reality. The blend of concepts involved, combined with a realistic project, engages students, encourages them to learn, and prepares them for their imminent professional or research activity. For this purpose, the $\equiv$ cart shown in Fig. 2(b)[Should this be Fig. 1(b)? w was robotized as shown in Fig. 3. The VEGO robotization presented here allows students to tackle a real case that includes mechanic design, choice of actuators, control architecture design, and integration of sensing systems, among other issues. Fig. 4 shows the architecture developed for controlling the autonomous vehicle. The architecture consists of four main elements: the control unit, acting unit, communication unit, and sensing unit, described below. The control software was developed using LabVIEW Robotics.

Control Unit: This is based on a programmable automation controller (PAC) CompactRIO by National Instruments, designed for applications that require high performance and reliability. It consists of: 1) a Freescale MPC5200 400-MHz processor with the Wind River VxWorks real-time operating system; 2) an FPGA (Xilinx) by which the processor has access
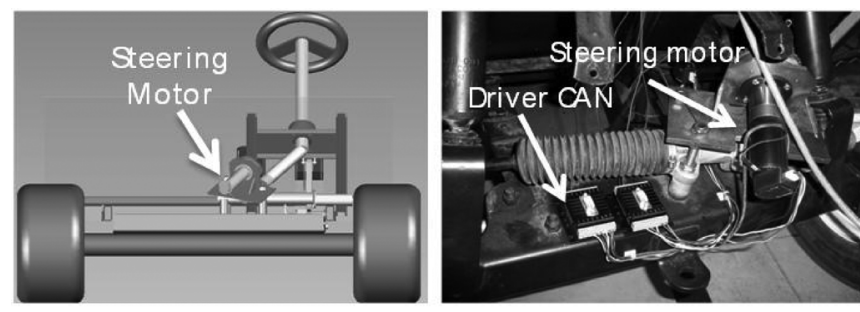

(a)
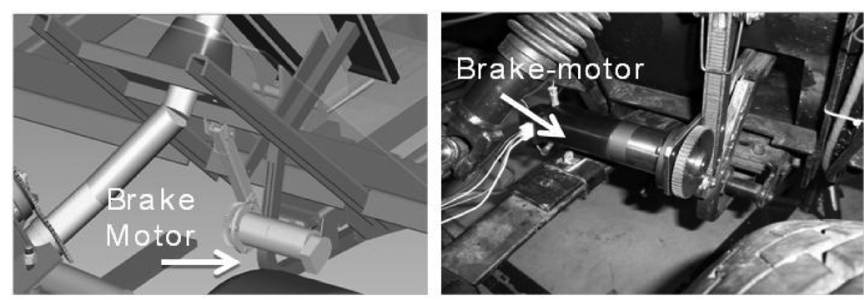

(b)
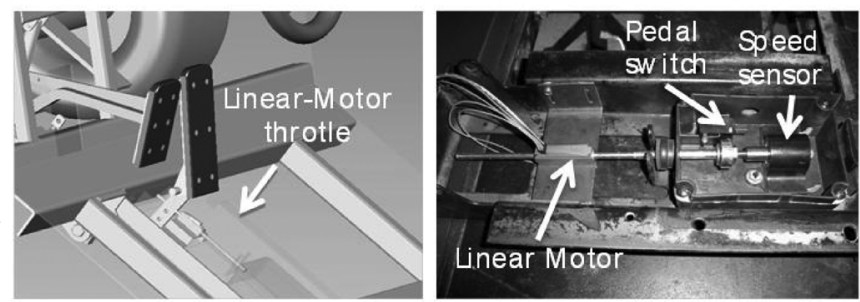

(c)

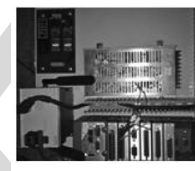

cRIO

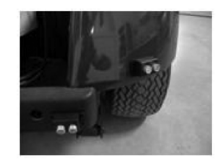

Ultrasound sensors

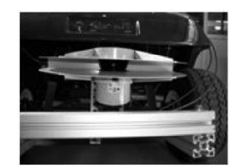

LIDAR (d)

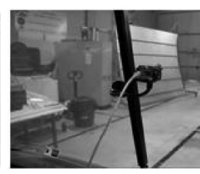

CCD Camera
Fig. 3. Robotized golf cart VEGO. (a) Steering modification. (b) Brake modification. (c) Throttle modification. (d) Control and sensor devices.

to I/O signals; and 3) various I/O modules allowing different typologies and protocols (digital, analog, CAN, RS485, Wireless, PROFIBUS, etc.).

Acting Unit: This allows the control unit to operate mechanically on the vehicle components (throttle, brake and steering) shown in Fig. 3. Each installed motor is provided with a CAN output driver connected to the communication unit of the vehicle.

Communication Unit cRIO: This communicates with the rest of the systems in the vehicle via several communication standards: 1) CANopen [12], used to send control orders to motors - three nodes were defined for this purpose, one for each component: throttle, brake and steering; 2) TCP/IP, used to exchange information with the laser telemetry system (LIDAR) installed in the vehicle, to receive movement orders in the teleoperated mode (via $\mathrm{Wi}-\mathrm{Fi}$ ), and as a means of communication with the programming station in the vehicle; and 3) RS232, used to receive the frames sent by the GPS installed in the vehicle.

Sensing Elements: The vehicle has the following sensors installed on-board: 1) LIDAR, with a measuring range of $20 \mathrm{~m}$ in $270^{\circ}$ with $0.25^{\circ}$ and $1-\mathrm{mm}$ precision, which generates a map of the environment in which the vehicle moves; 2) an ultrasound ring with eight sensors that allows obstacles to be detected at a distance of up to $2 \mathrm{~m}$; 3 ) a GPS and gyroscope that give the 


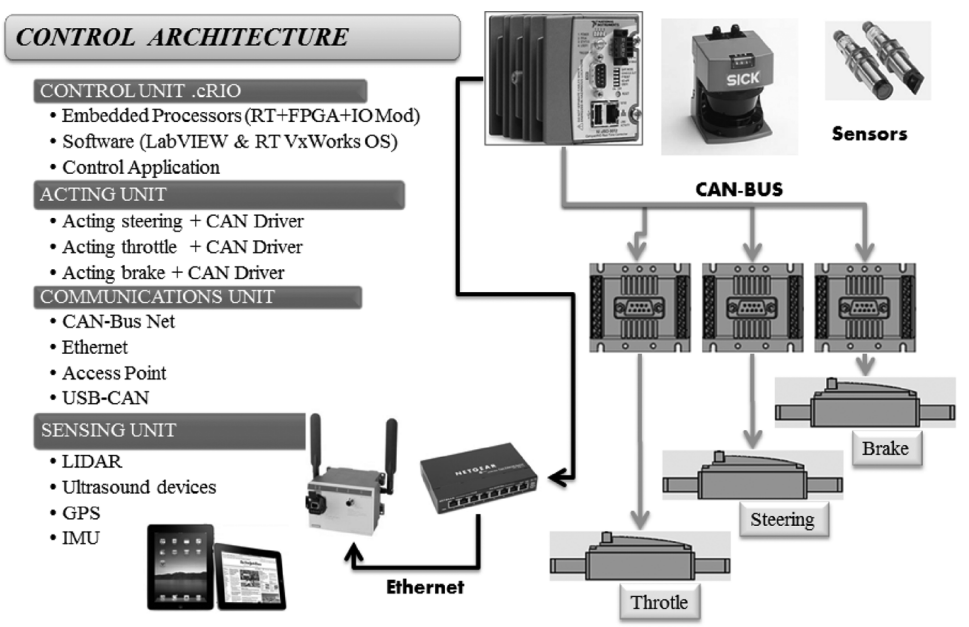

Fig. 4. VEGO control architecture.

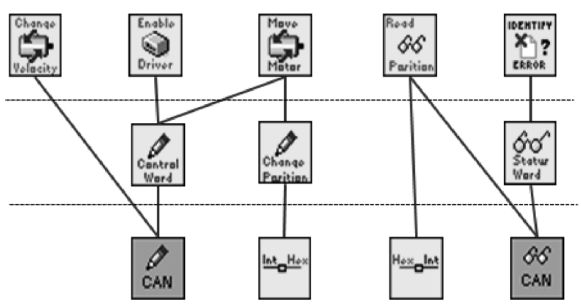

(a)

Fig. 5. Examples of developed libraries. (a) Motor control library hierarchy. (b) LIDAR control library structure.

Fig. 5. Examples of developed libraries. (a) Motor control library hierarchy. (b) LIDAR control library structure.

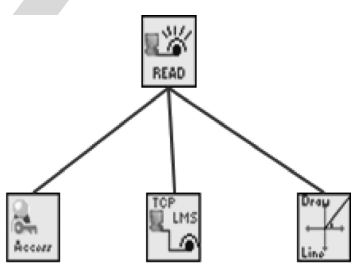

(b)

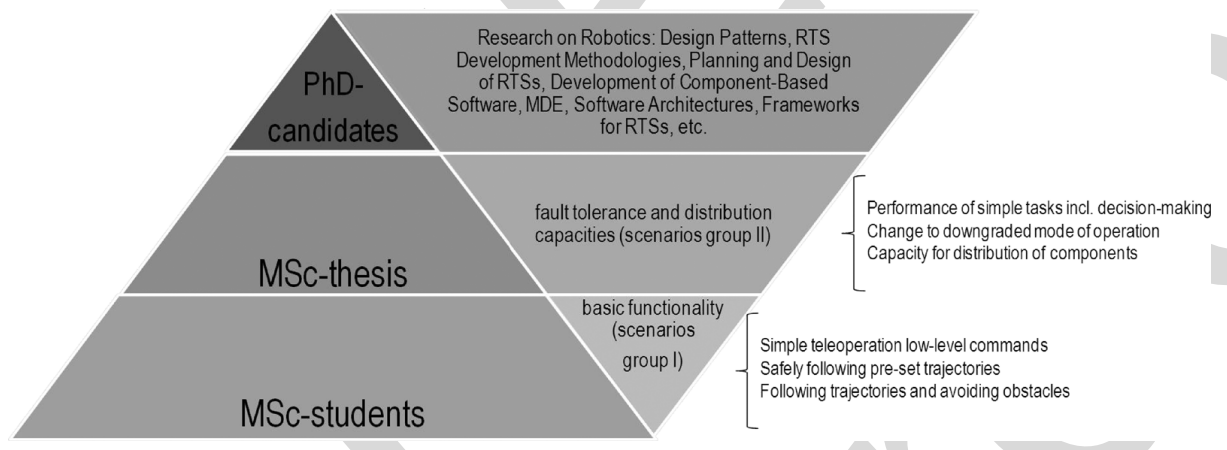

Fig. 6. Types of students and their use of the platform.

global and local position at any moment; and 4) a vision system that allows detection and classification of objects encountered in front of the vehicle (people, traffic signs, and the like).

Control Software: The algorithm implementation for vehicle control was carried out using LabVIEW. Various libraries were developed for the functions used for controlling the vehicle. Fig. 5 shows two examples of these libraries. One of them, whose structure is depicted in Fig. 5(a), contains the functions for motor communication via CANopen. The functions implement low-level commands allowing communication via the CAN interface between the control system and motor drivers in order to control the vehicle trajectory. Fig. 5(b) shows the structure of the library developed for the LIDAR Sick-LMS200 control and its communication with the cRIO control unit.

\section{LAB MODULES}

The VEGO robotized autonomous vehicle represents a real platform intended for solving scenarios with increasingly complex tasks that require the incorporation of additional functionalities. VEGO is used by all students, as shown in Fig. 6: those only enrolled in the Robotics module, those who use the robot for their M.Sc. thesis work, and those who continue to their doctoral thesis. Students begin with very simple tasks that are made more and more complex by adding new components and interactions.

The scenarios are structured in two groups, as shown in Table I:

- Group I: The objective of these scenarios is to provide students with a framework within which they can continuously validate the work carried out in the classroom. The internal complexity of the tasks is kept to a minimum (they can be implemented in only a few weeks using traditional techniques) since the objective is not to test algorithms, but to provide support as described. Despite their simplicity, these scenarios will make it possible to validate all aspects of the material taught in the Robotics module. 


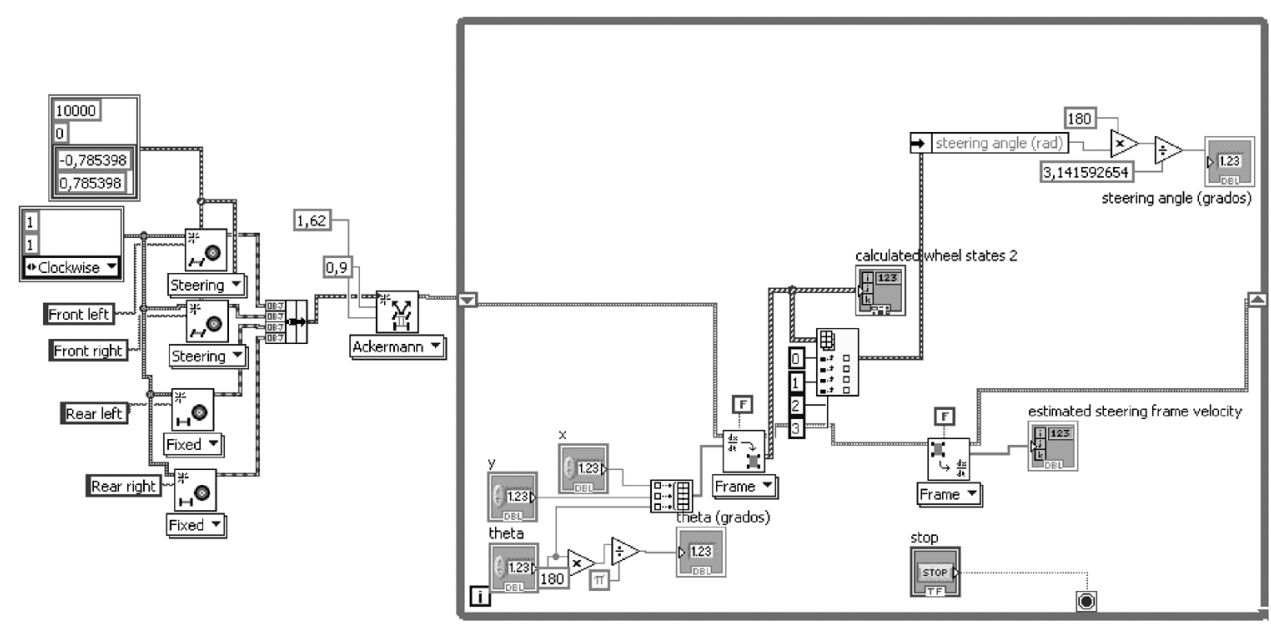

Fig. 7. LabVIEW application for VEGO's teleoperation.

TABLE I

INCREASINGLY COMPLEX SCENARIOS FOR ALL STUDENTS USING THE ROBOTIC VEHICLE

\begin{tabular}{|c|c|c|c|}
\hline \multirow[b]{2}{*}{ ڤั) } & $\begin{array}{l}\text { Implement simple } \\
\text { teleoperation (low-level } \\
\text { commands) }\end{array}$ & $\begin{array}{l}\text { Implement safely following pre- } \\
\text { set trajectories }\end{array}$ & $\begin{array}{l}\text { Implement the following of } \\
\text { trajectories and avoiding } \\
\text { obstacles } \\
\end{array}$ \\
\hline & $\begin{array}{l}\text { Capacity to enter simple } \\
\text { commands (start up, stop, set } \\
\text { speed, etc.). Capacity to } \\
\text { control motors and actuators. } \\
\text { No sensory capacity. }\end{array}$ & $\begin{array}{l}\text { Capacity to enter polygonal } \\
\text { trajectories. Graphic representation } \\
\text { of vehicle status and trajectory. } \\
\text { Conversion of trajectories to low- } \\
\text { level commands. Capacity to react to } \\
\text { obstacle warning (emergency stop). } \\
\text { Capacity to detect obstacles. } \\
\text { Controller-ultrasound.GUI- } \\
\text { ultrasound. GUI-controller. }\end{array}$ & $\begin{array}{l}\text { Capacity to display the vehicle } \\
\text { and the environment in which it } \\
\text { moves. Capacity to avoid } \\
\text { cylindrical obstacles and resume a } \\
\text { straight trajectory. Capacity to } \\
\text { identify contours. Controller- } \\
\text { odometrics. Controller-telemetry. } \\
\text { GUI-odometrics. GUI-telemetry }\end{array}$ \\
\hline \multirow[b]{2}{*}{$\frac{\overline{0}}{\bar{\Xi}}$} & $\begin{array}{l}\text { Performance of simple } \\
\text { tasks including decision- } \\
\text { making }\end{array}$ & $\begin{array}{l}\text { Change to downgraded mode of } \\
\text { operation }\end{array}$ & $\begin{array}{l}\text { Capacity for distribution of } \\
\text { components }\end{array}$ \\
\hline & $\begin{array}{l}\text { Capacity to define simple } \\
\text { tasks. Expanded GUI. Making } \\
\text { simple decisions to achieve an } \\
\text { objective. Map generation. } \\
\text { Capacity to interpret data from } \\
\text { the environment. Maps of } \\
\text { environment. Mission control } \\
\text { agents. Capacity to recognize } \\
\text { visual references. Vision } \\
\text { system. Controller-maps. } \\
\text { Controller-vision. Maps- } \\
\text { vision. GUI-vision }\end{array}$ & $\begin{array}{l}\text { Capacity to detect failures in the } \\
\text { odometrics system. Capacity to } \\
\text { reconFig. and switch to downgraded } \\
\text { mode. Odometrics monitor. } \\
\text { Odometer Hw failure detector. } \\
\text { Controller-odometer-monitor. }\end{array}$ & $\begin{array}{l}\text { Distribution capacity. GUI- } \\
\text { ultrasound. GUI-controller }\end{array}$ \\
\hline
\end{tabular}

- Group II: This group of scenarios marks a qualitative change in terms of both complexity and objectives. They will be carried out as a part of the M.Sc. thesis since they require a substantial advance in the student's ability in order to be able to meet these new challenges. They essentially serve to test patterns of failure tolerance and distribution. More complex functionalities (for instance, map generation) will be implemented on the basis of already-available commercial or free components.

Two examples of applications from different scenarios resolved by students are shown as follows. Fig. 7 displays an application that permits VEGO to be teleoperated to perform linear or curvy trajectories, corresponding to Group I's scenario in Table I, "Implement simple teleoperation"; in this case, vehicle safety relies on ultrasound sensors. Fig. 8 shows an application that allows the generation of a 2-D map of the environment using the LIDAR in addition to generating and following trajectories, corresponding to Group II's scenario in Table I, "Performance of simple tasks including decision-making." For the vehicle navigation, an avoiding-obstacle algorithm known as Vector Field Histogram (VFH) [13] was implemented. Fig. 8(d) shows the navigation interface designed to allow missions in semi-structured environments. Once the environment has been chosen for the mission, the planner divides the mission into lower complexity missions. Such submissions are supplied to the VFH to have it calculate the vehicle trajectories at each moment. Mission maps are loaded in two files: one in JPG format corresponding to the environment image and another with configuration data. The data include the GPS position of the core of the image, the image size in pixels, and a zoom of the picture when it was taken. The route planning is fixed by the user by marking the initial and final point of the mission on the preloaded image of the environment. Then, the planner selects the optimal route by 


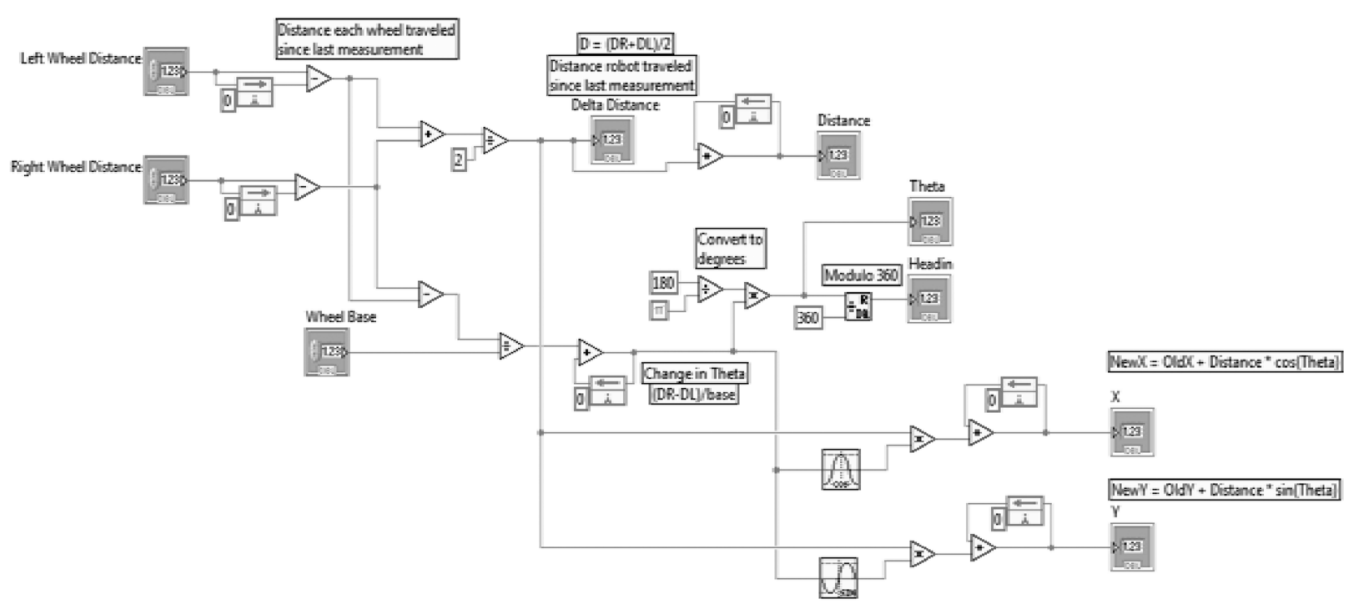

(a)

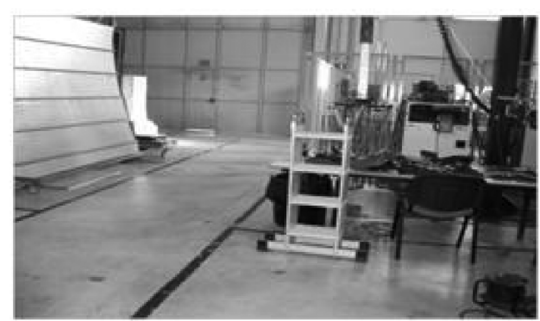

(b)

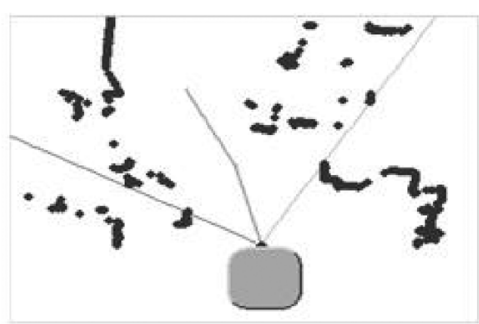

(c)

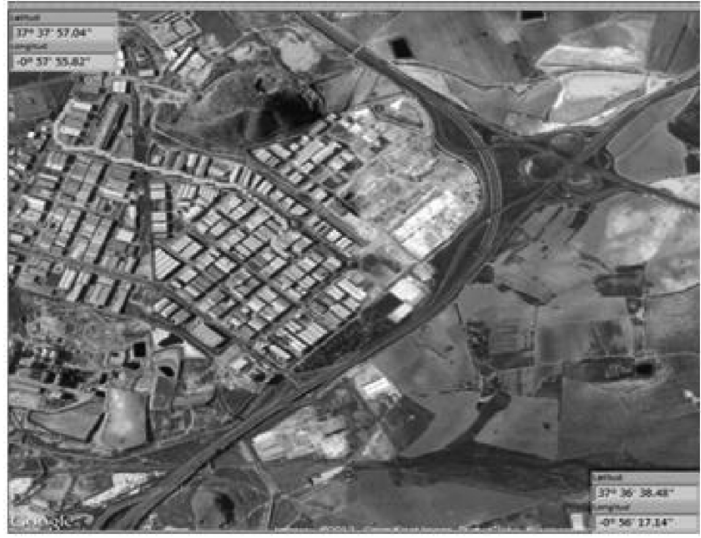

(d)

Fig. 8. VEGO's simple mission execution. (a) Part of the LabVIEW code for mission execution: odometry calculation. (b) Front view image from LIDAR perspective. (c) Generated polygonal trajectory. (d) VEGO's navigation interface (light line shows an outdoor trajectory).

means of the algorithm A* [14]. Finally, the route is executed, aided by on-board sensors (LIDAR and Vision System).

\section{Evaluation of the Proposal}

During the last three academic years of the M/SC.-ICT (2008-2009 to 2010-2011), the lecturers conducted surveys to elicit the evolution of students' perceptions of their previous experience in topics directly related to the Robotics module. Only the above-mentioned 27 students who carried out their M.Sc. thesis using robotized vehicles took part in the survey willingly (7, 9, and 11 students per course offering). The reference point for this study comes from the IEEE-CS/ACM curricular recommendation for Computer Science (CS2008) [15]. This curriculum seeks, among other objectives, to identify the fundamental skills and knowledge that all computing students must possess. The CS2008 curriculum defines 14 Knowledge
Areas divided into Knowledge Units, and within those are Knowledge Topics. The areas of knowledge selected for their marked influence on robotics were Programming Fundamentals, Operating Systems, Graphics and Visual Computing, and Intelligent Systems.

The four knowledge units most closely related to robotics were selected from each knowledge area. For example, the knowledge units chosen from Intelligent Systems were Basic Search Strategies, Planning Systems, Robotics, and Perception. In order to facilitate decision-making for respondents, each knowledge area was described by the detailed knowledge topics and learning objectives that allow the contextualizing of topics within each knowledge unit since they are intended to capture what students are able to do with this knowledge. For example, the knowledge topics for the knowledge unit of Robotics were Planning vs. Reactive Model, Sensing, Robot Programming, 


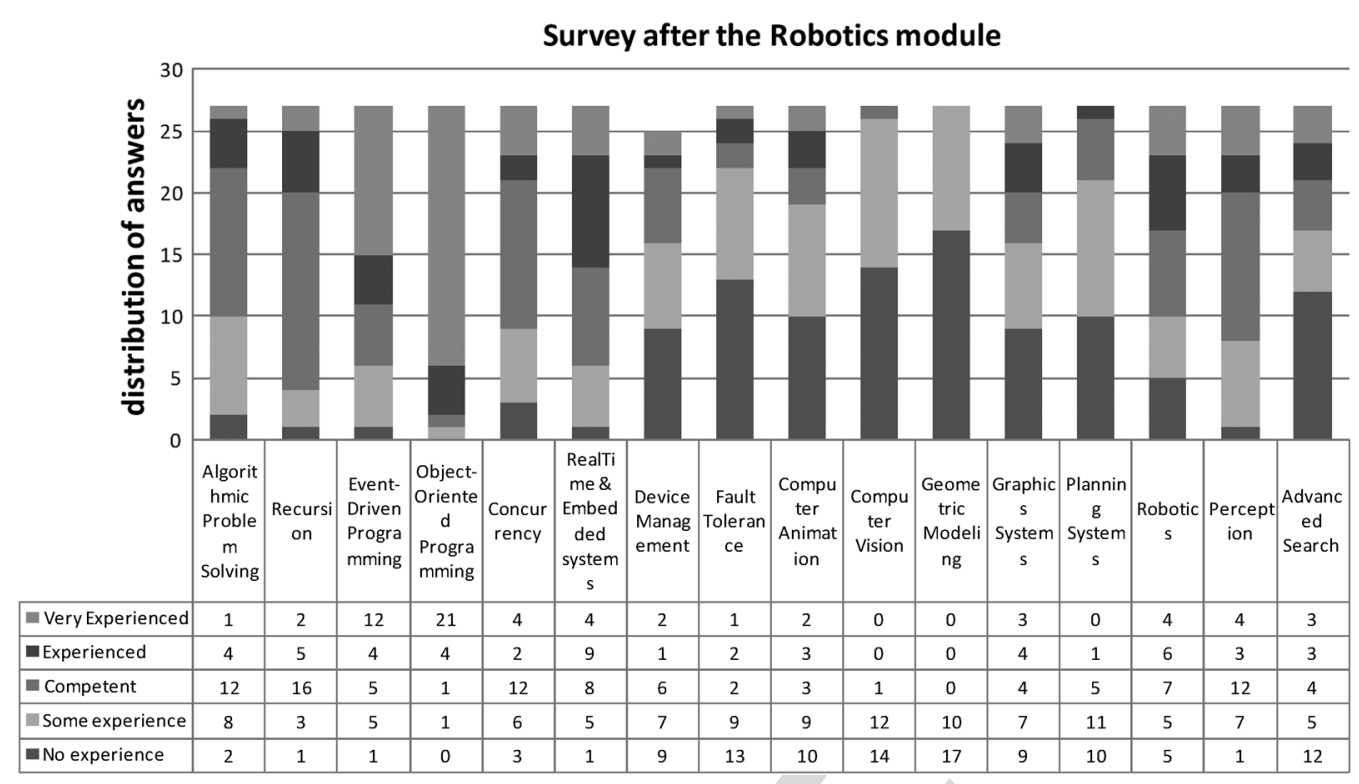

Fig. 9. Results of the survey after finishing the Robotic module. The lower part shows the numerical distribution of the answers.

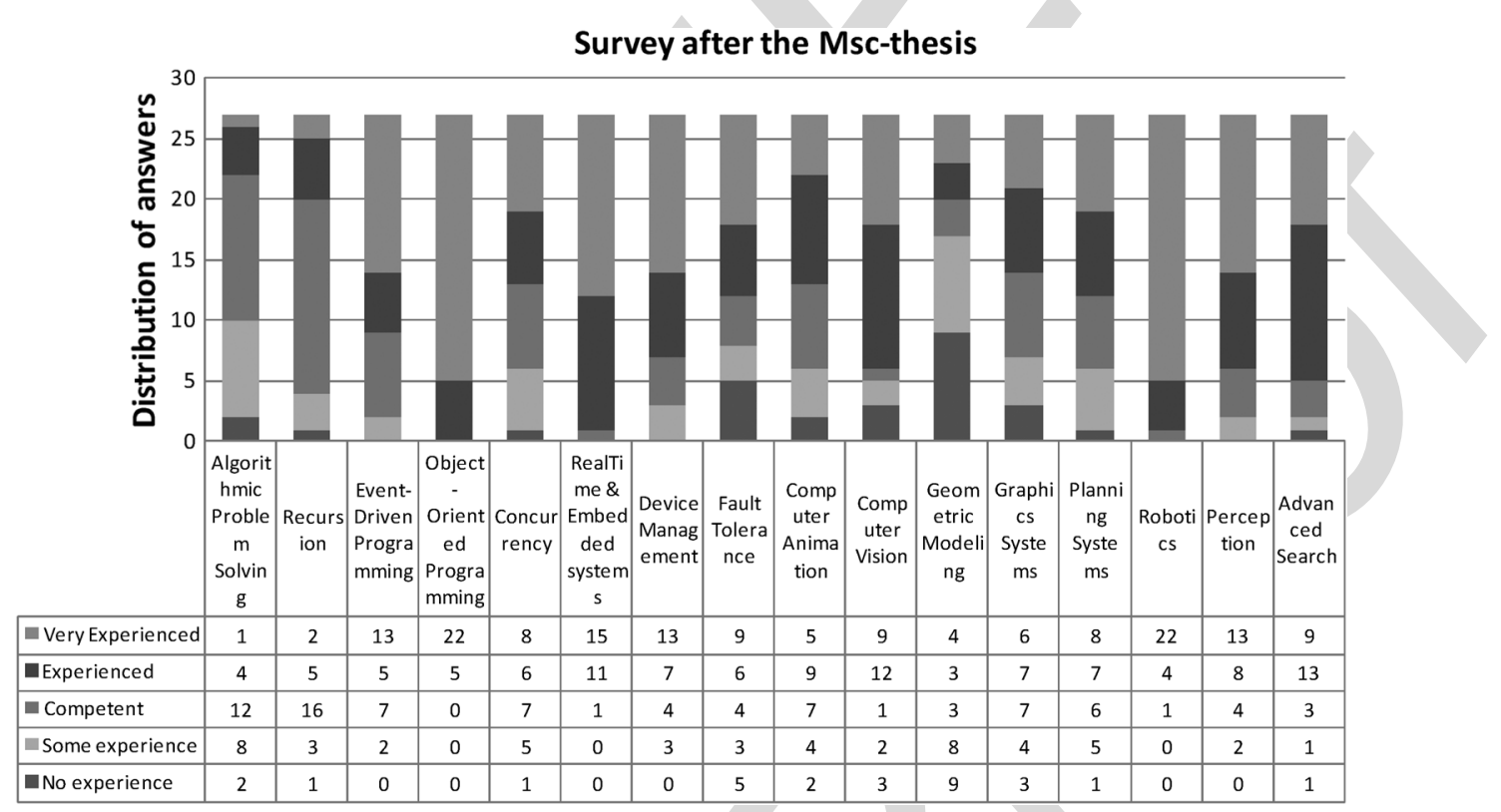

Fig. 10. Results of the survey after using the robotized vehicle.

Robot Software and Architecture, Navigation and Control. Then, a questionnaire was administered that had $4 \times 4=16$ questions, which students had to answer by indicating their perception about the training level for each knowledge unit. A five-point Likert scale was used, with a range of answers from " 1 -No experience" to "5-Very experienced."

The survey was carried out twice: first, after finishing the Robotics and Computer Vision teaching period, and second, after finishing the M.Sc. thesis using VEGO. This comparison revealed the students' perception after finishing their training with the use of a real platform like that described here; see Fig. 9. As observed, students are positive with respect to their training in event and object-oriented programming. Although they consider this acceptable in topics directly related to robotics, they did not find it totally satisfactory after completing the robotics module. Fig. 10 shows the evolution of their perception of the same group of students after finishing their M.Sc. theses six months later. There is a significant increase with respect to the knowledge units most closely connected to robotics (planning systems, computer vision, robotics, real-time and embedded systems, device management, perception) as a result of the availability of an integrated platform with multidisciplinary possibilities. The number of students who perceive their level of training as "very experienced" $(13.25 \%)$ is significantly increased in comparison to the previous situation (3.93\%). Likewise, the percentage of students who considered themselves as not having any experience $(6.75 \%)$ is reduced to $1.07 \%$. 


\section{CONCLUSION}

A novel approach for teaching robotics at the postgraduate level has been presented here. The approach consists of the use of industrial-like vehicle platforms with the aim of improving learning opportunities for postgraduate students; the impact of the proposed approach was carefully evaluated with two surveys per year. The platforms described expose students to hands-on learning, which has led to promising results in the topics of algorithmic problem solving, recursion, fault tolerance, concurrence and advanced s $\equiv$. The trend seems to be that the more

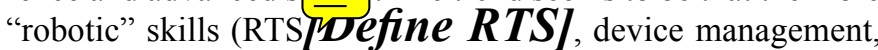
robotics, perception, computer vision, and planning systems) are really favored by the proposed approach. Nevertheless, in the case of particular learning objectives like computer animation, geometric modeling, and graphic systems, students' perception has not improved so markedly. The experience gained from implementing these various scenarios is expected, in the medium term, to lead to new case studies that will also contribute to improving learning.

\section{REFERENCES}

[1] R. Edlinger and M. Zauner, "A modular and scalable electronic system for mobile and autonomous robots," in Lecture Notes in Communications in Computer and Information Science (CCIS). New York: Springer Verlag, 2010, p. 9.

[2] M. T. Chew, S. Demidenko, G. S. Gupta, and L. Huang, "Mobile robots in the engineering education setting," in Proc. 5th Int. Conf. Autom., Robot. Appl., Wellington, New Zealand, Dec. 2011, pp. 1-6.

[3] R. Dhaouadi and M. A. Sleiman, "Development of a modular mobile robot platform: Applications in motion-control education," IEEE Ind. Electron. Mag., vol. 5, no. 4, pp. 35-45, Dec. 2011.

[4] J. M. Gómez-De-Gabriel, A. Mandow, J. Fernández-Lozano, and A. J. García-Cerezo, "Using LEGO NXT mobile robots with Lab VIEW for undergraduate courses on mechatronics," IEEE Trans. Educ., vol. 54, no. 1, pp. 41-47, Feb. 2011.

[5] L. C. McNinch, R. A. Soltan, K. R. Muske, H. Ashrafiuon, and J. C. Peyton Jones, "An experimental mobile robot platform for autonomous systems research and education," in Proc. IASTED Int. Conf. Robot. Appl., Cambridge, MA, Nov. 2009, pp. 412-418.

[6] C. Cardeira and J. S. Da Costa, "A low cost mobile robot for engineering education," in IECON Proc., Raleigh, NC, Nov. 2005, pp. $2162-2167$.

[7] L. Greenwald and J. Kopena, "Mobile robot labs," IEEE Robot. Autom. Mag., vol. 10, no. 2, pp. 25-32, Jun. 2003.

[8] Y. Y. Ugurlu, "Project-based learning using LabVIEW and embedded hardware," in Proc. IEEE/SICE SII, Tokyo, Japan, Dec. 2011, pp. 561-566.
[9] M. Barak and Y. Zadok, "Robotics projects and learning concepts in science, technology and problem solving," Int. J. Technol. Design Educ., vol. 19, no. 3, pp. 289-307, Aug. 2009.

[10] R. Jamal, "Requirements and Solutions in Applied Robotics," in Research and Education in Robotics-EUROBOT 2010-Communications in Computer and Information Science. Berlin, Germany: Springer-Verlag, 2011, vol. 156, pp. 87-92.

[11] R. J. Gross and P. Y. Oh, "A scalable software toolkit fo humanoids," in Proc. ARCS, Orlando, FL, Jul. 2010[Paḡa?].

[12] H. Boterenbrood, "CANopen: High level protocol for CAN-bus," 2000 [Online]. Available: http://www.nikhef.nl/pub/departments/ct/po/doc/ CANopen30.pdf

[13] Y. Cang, "Navigating a mobile robot by a traversability field histogram," IEEE Trans. Syst. Man. Cybern. B, Cybern., vol. 37, no. 2, pp. 361-372, Apr. 2007.

[14] P. Lester, "A* pathfinding for beginners," 2005 [Online]. Available: http://www.policyalmanac.org/games/aStarTutorial.htm

[15] ACM, IEEE-CS, "Computer science curriculum 2008: An interim revision of CS 2001," 2008 [Online]. Available: http://www.acm. org/education/curricula/ComputerScience2008.pdf

Pedro J. Navarro graduated in Electrical and Electronics Engineering and received the Ph.D. degree in computer vision from the Technical University of Cartagena, Cartagena, Spain in 2001 and 2009, respectively.

In 2001, he joined the Department of Technologies of Information and the Communications, Technical University of Cartagena, where he has been an Associate Professor since 2003. His current research interests includes automated visual inspection systems, image processing techniques for detect defects in texture, and advanced robotics and intelligent transportation systems, with special emphasis on computer vision.

Carlos Fernández received the M.S. degree in industrial engineering and Ph.D. degree in artificial intelligence and robotics from the Technical University of Madrid, Madrid, Spain.[Years degrees were received?]

$\mathrm{He}$ is an Associate Professor of computer science with the Technical University of Cartagena, Cartagena, Spain. He was with the Technical University of Madrid, where he became an Associate Professor of control in 1993. In 2000, he joined the Systems and Electronic Engineering Division (DSIE), Technical University of Cartagena. Since 1990, he has participated in several research and development projects for the development of machine vision and robotic systems for various industrial sectors. His current research interests are in computer vision, software reusability, distributed systems, and robotics.

Pedro Sánchez received the Ph.D. degree in computer science from the Technical University of Valencia, Valencia, Spain, in 2000.

Since 1996, he has participated in various projects focused on software engineering and conceptual modeling applied to the development of reactive systems. In 2000, he joined the Systems and Electronic Engineering Division (DSIE), Technical University of Cartagena, Cartagena, Spain. He is currently an Associate Professor with the Technical University of Cartagena in the field of computer science. His current research interests include model-driven engineering and real-time systems. 